\title{
Preparação e Avaliação Reacional de Nanocompósitos de PVP K-30 - Montmorilonita (Natural e Organicamente Modificada) por Difração de Raios X
}

\author{
Camila B. Dornelas, Daniel K. Resende, Maria Inês B. Tavares, Ailton S. Gomes \\ Instituto de Macromoléculas Professora Eloisa Mano, UFRJ \\ Lúcio M. Cabral \\ Departamento de Ciências Farmacêuticas, UFRJ
}

\begin{abstract}
Resumo: Em estudo anterior foi utilizado o conceito de nanocompósito para aplicações farmacêuticas, mais especificamente na liberação controlada de fármacos. Um nanocompósito polímero (PVP K-30) - silicato lamelar (argila organofílica, OMMT) foi preparado por solução, em diclorometano, e a sua avaliação como excipiente farmacêutico foi realizada com sucesso. Neste trabalho, um estudo do tempo reacional foi realizado (12, 48 e 72 horas), tendo sido observado, através de difração de raios X (DRX), um valor de espaçamento interlamelar máximo em 12 horas. Este resultado motivou um estudo mais detalhado a respeito deste processo de intercalação. Para tal, e em razão da solubilidade da PVP, foi avaliado, também, um sistema mais simples, com a argila sódica (MMT), não tratada, em água. Em ambos os sistemas, PVP-OMMT e PVP-MMT, foi realizada uma varredura de tempos reacionais (de 15 minutos a 72 horas), nas proporções de 2:1, 1:1 e 1:2, para caracterização por DRX. As análises sugerem a formação dos nanocompósitos em até 1 hora de reação, resultado atribuído à estrutura amídica da PVP, capaz de estabilizar as lamelas negativamente carregadas da argila.
\end{abstract}

Palavras-chave: PVP K-30, argila sódica, argila organofílica, nanocompósito.

Preparation and Reactional Evaluation of Formation of PVP K-30 - Montmorillonite (Natural and Organophilic) by X Ray Diffraction

Abstract: In a previous study the concept of nanocomposite for pharmaceutical applications, more specifically in drugs' release, was used. A polymer-layered silicate nanocomposite (PLN) of PVP K-30 - organophilic MMT (OMMT) at a $2: 1$ ratio was produced in dichloromethane solution and successfully evaluated as a pharmaceutical adjuvant. In this study the influence of reaction time was investigated. XRD data indicates that maximum interlamellar distances were achieved after 12 hours. These results lead us to carry out a more detailed study in order to follow the intercalation process. The influence of several increasing reaction times (15 minutes to 72 hours) on the interplanar distances of PVP-OMMT (in dichloromethane solution) and PVP-MMT (in aqueous solution) at 2:1, 1:1 and 1:2 ratios was investigated with XRD. The data obtained showed that new PLN were obtained at short time reaction times (up to 1 hour), which was attributed to amide structure of the polymer (PVP), capable of establishing links with the anionic clay lamellae.

Keywords: PVP K-30, MMT, OMMT, PLN.

\section{Introdução}

A nanotecnologia está relacionada às estruturas, propriedades e processos envolvendo materiais com dimensões numa escala de 1 a $300 \mathrm{~nm}$, valores estes, considerados elevados quando comparados a moléculas simples, porém pequenos em relação ao comprimento de onda da luz visível ${ }^{[1]}$. Dentre os materiais nanoestruturados podem ser encontrados nanopartículas, nanocristais, nanofios, nanotubos, nanocompósitos. O grande diferencial destes materiais é a potencialização de propriedades físicas e químicas dos produtos obtidos a partir dos mesmos. Enquanto alguns materiais naturais de estruturas nanométricas são empregados comercialmente há muitos anos, os nanomateriais manufaturados foram recentemente reconhecidos como uma nova classe de materiais, sendo que alguns tipos têm demonstrado particularidades interessantes e propriedades promissoras, em especial na área farmacêutica.

O conceito de nanotecnologia em aplicações farmacêuticas, mais especificamente na liberação de fármacos, está baseado na produção de nanopartículas que contêm moléculas do fármaco a serem depositadas exclusivamente no órgão alvo com a vantagem de serem inativas. Neste contexto, destaca-se a busca por sistemas terapêuticos capazes de modular de forma satisfatória a cinética, o tempo e o local de liberação de um fármaco, minimizando ao mesmo 
tempo, os efeitos colaterais a eles associados. Isto pode ser obtido através da liberação gradual de substâncias ativas veiculadas nos chamados sistemas de liberação modificada ${ }^{[2-5]}$.

A polivinilpirrolidona (PVP), também chamada de povidona (Figura 1), é um homopolímero de N-vinil-2-pirrolidona obtido por polimerização via radicalar em água ou em álcool isopropílico, de natureza higroscópica e compatível com uma ampla faixa de resinas hidrofílicas e hidrofóbicas ${ }^{[6,7]}$. É um pó branco amorfo de fluxo livre, solúvel tanto em água quanto em solventes orgânicos. O peso molecular da PVP é freqüentemente relatado por K - valor de Fikentscher, derivado da viscosidade de uma solução $0^{[8]}$. A relação entre a viscosidade intrínseca, o valor $\mathrm{K}$ e o peso molecular aproximado para a PVP é ilustrada pela Tabela ${ }^{[9]}$. Neste estudo foi feito uso da PVP K-30.

Este polímero é bastante difundido na tecnologia farmacêutica ${ }^{[10,11]}$, em especial, na modulação da dissolução de fármacos pouco solúveis em água. Uma das abordagens clássicas em termos de aumento da dissolução de fármacos pouco solúveis em água está na maximização da estrutura porosa de uma matriz polimérica solúvel em água ${ }^{[12]}$, como é o caso da PVP. O objetivo do recurso é aumentar a superfície de contato do fármaco pouco solúvel em água com o meio dissolutor.

A utilização de argilas já é bastante descrita na composição de formulações farmacêuticas ${ }^{[13-15]}$. A bentonita é uma substância com propriedades que são requeridas para utilização como excipiente funcional em comprimidos, visto a sua capacidade de formar géis em concentrações baixas por intumescimento em água, sendo apropriado seu uso como agente ligante e desintegrante. A bentonita é uma argila plástica resultante de alterações de cinzas vulcânicas, constituída predominantemente de montmorilonita, uma espécie de argila natural do grupo esmectita descoberta em 1847 na França (Montmorillon) por Damour e Salvetat ${ }^{[16]}$. A montmorilonita é um filossilicato lamelar do tipo 2:1 (2 tetraedros de silício: 1 octaedro de alumínio), de fórmula geral $\left[\mathrm{M}_{\mathrm{x}}\left(\mathrm{Al}_{4-\mathrm{x}} \mathrm{Mg}_{\mathrm{x}}\right)\right.$ $\mathrm{Si}_{8} \mathrm{O}_{20}(\mathrm{OH})_{4}$, onde $\mathrm{M}$ é um cátion monovalente e $\mathrm{x}$, o grau de substituição isomórfica $(0,5-1,3)$. Em relação a sua micro-

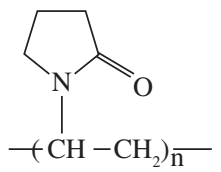

Figura 1. Estrutura química da PVP.

Tabela 1. Relação entre viscosidade, $K$ e peso molecular $\left(M_{n}\right.$ e $\left.M_{w}\right)$ para a $\mathrm{PVP}{ }^{[9]}$

\begin{tabular}{ccrr}
\hline $\begin{array}{c}\text { Viscosidade em } \\
\text { água cSt (PVP\%) }\end{array}$ & $\begin{array}{c}\text { Escala de } \\
\text { valor K }\end{array}$ & \multicolumn{1}{c}{$\mathbf{M}_{\mathbf{n}}$} & $\mathbf{M}_{\mathbf{w}}$ \\
\hline $7(20)$ & $13-19$ & 10.000 & 12.000 \\
$25(20)$ & $26-34$ & 40.000 & 55.000 \\
$50(10)$ & $50-62$ & 220.000 & 400.000 \\
$400(10)$ & $80-100$ & 630.000 & 1.280 .000 \\
$7000(10)$ & $115-125$ & 1.450 .000 & 2.800 .000 \\
\hline
\end{tabular}

estrutura, a lamela apresenta um diâmetro aproximado entre 100 a $200 \mathrm{~nm}$ e $1 \mathrm{~nm}$ de espessura. De cinco a dez lamelas estão associadas pela presença de íons entre essas na partícula primária (posição transversal de 8 a $10 \mathrm{~nm}$ ), as quais formam grandes agregados irregulares (diâmetro de 0,1 a $10 \mu \mathrm{m}$ ) levando a uma estrutura estratificada na argila ${ }^{[17,18]}$.

Uma alternativa à bentonita seria o viscogel $\mathrm{B} 8$, uma argila modificada organicamente pela inserção de sal de octadecilamina, que combinaria a capacidade de intumescimento, apesar de reduzida, com a ação tensoativa do sal de alquilamônio ${ }^{[19]}$. Os cristais das argilas têm uma carga negativa permanente em decorrência de rearranjos iônicos isomórficos lamelares, os quais são compensados por cátions hidratados em solução aquosa. O tratamento das argilas com moléculas orgânicas catiônicas resulta em uma superfície hidrofóbica, que leva à modificação de inúmeras de suas propriedades iniciais ${ }^{[20]}$. Estes silicatos são usualmente conhecidos como organoargilas.

\section{Experimental}

\section{Intercalação polímero-argila}

Há inúmeras estratégias utilizadas para o preparo de nanocompósitos polímero-silicato lamelar ${ }^{[21]}$, a intercalação em questão seguiu o processo de intercalação-adsorção de polímeros em solução, através do qual o silicato lamelar é intercalado entre suas lamelas pelo material polimérico na presença de um solvente no qual o polímero seja solúvel. Em razão das forças que mantêm as lamelas "empilhadas" serem fracas, tais silicatos lamelares podem ser facilmente afastados com o uso de um solvente adequado. O polímero, então, permeia no espaço interlamelar e, quando o solvente é evaporado (ou a mistura precipitada), as lâminas se reorganizam estando agora alternadas pela presença de polímero para formar, na melhor das hipóteses, uma estrutura multilamelar ordenada. Para fins comparativos, foram realizados dois estudos, a intercalação da PVP na organoargila de nome comercial viscogel B8 e na bentonita natural, ou argila sódica.

A intercalação do polímero com o viscogel B8 (OMMT) foi conduzida da seguinte maneira:

- Foram pesadas quantidades suficientes de PVP K-30 e de viscogel B8, de modo que se mantivessem as proporções de 2:1, 1:1 e 1:2 entre eles;

- O sistema foi deixado sob agitação por diferentes tempos reacionais (15 minutos, 30 minutos, 45 minutos, 1 hora, 24 horas, 48 horas, 72 horas) em diclorometano a temperatura ambiente; e

- A remoção do solvente foi feita por pressão reduzida em rotaevaporador, a uma temperatura de $45-50{ }^{\circ} \mathrm{C}$.

A intercalação do polímero com a argila sódica (MMT) foi conduzida da seguinte maneira:

- Foram pesadas quantidades suficientes de PVP K-30 e de argila sódica, de modo que se mantivessem as proporções de 2:1, 1:1 e 1:2 entre elas; 
- O sistema foi deixado sob agitação por diferentes tempos reacionais (15 minutos, 30 minutos, 45 minutos, 1 hora, 24 horas, 48 horas, 72 horas) em água a temperatura ambiente; e

- A remoção do solvente foi feita através de centrifugação a 4000 rpm.

Vale ressaltar que, em razão da organofilia do viscogel B8, utilizou-se como solvente o diclorometano, escolhido por ter assegurada rapidamente a sua remoção sob condições brandas. Aproveitando-se da natureza hidrofílica da argila sódica, optou-se pela água, solvente universal e de custo reduzido, cabendo, então, a necessidade de outro procedimento de remoção do solvente que não o rotaevaporador, o que justifica a escolha pela centrifugação.

\section{Caracterização - difração de raios X (DRX)}

As amostras foram analisadas em Difratômetro de raios $\mathrm{X}$ operado a $40 \mathrm{kV}$ e $30 \mathrm{~mA}$. O ângulo de difração $2 \theta$ foi registrado de 2 a $20^{\circ} \mathrm{em}$ temperatura ambiente e a radiação $\mathrm{CuK} \alpha$ foi utilizada como fonte dos raios X. Para obtenção do valor de espaçamento interlamelar foi realizado tratamento matemático e aplicação da Equação de Bragg ${ }^{[20]}$.

\section{Resultados e Discussão}

\section{PVP K-30 - OMMT}

Os difratogramas de raios $\mathrm{X}$ dos sistemas polímero - organoargila, na proporção 2:1, em todos os diferentes tempos reacionais testados (15 minutos, 30 minutos, 45 minutos, 1 hora, 24 horas, 48 horas, 72 horas) em diclorometano a temperatura ambiente encontram-se na Figura 2. Os valores de espaçamento interlamelar correspondentes podem ser acompanhados pela Tabela 2.

Como previsto, devido ao grande volume de massa ocupado pelo polímero, foi notado um aumento significativo do espaçamento interlamelar, superior a $5 \AA$, em comparação com o viscogel B8 puro $(25,98 \AA$ ), evidenciando a formação do nanocompósito. O interessante é observar que com 45 minutos de reação é alcançada a distância interlamelar

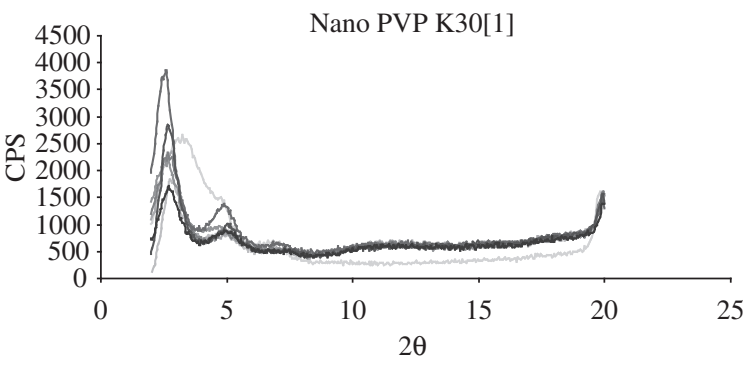

$$
\begin{gathered}
\hline \text { viscogel B8 }- \text { 2p1a } 15 \text { minutos }- \text { 2p1a } 30 \text { minutos } \\
- \text { 2p1a } 45 \text { minutos - 2p1a } 1 \text { hora }-2 \text { p1a } 24 \text { horas } \\
- \text { 2p1a } 48 \text { horas }- \text { 2p1a } 72 \text { horas }
\end{gathered}
$$

Figura 2. Difratogramas dos sistemas polímero - organoargila estudados, na proporção 2:1.
Tabela 2. Valores de espaçamento interlamelar calculados para os sistemas polímero - organoargila estudados, na proporção 2:1.

\begin{tabular}{lcc}
\hline $\begin{array}{c}\text { Tempo } \\
\text { reacional }\end{array}$ & Deslocamento $\left(^{\circ}\right)$ & $\begin{array}{c}\text { Espaçamento } \\
\text { interlamelar }(\AA)\end{array}$ \\
\hline Viscogel B8 & 3,40 & 25,98 \\
15 minutos & 2,85 & 31,00 \\
30 minutos & 2,80 & 31,56 \\
45 minutos & 2,70 & 32,72 \\
1 hora & 2,70 & 32,72 \\
24 horas & 2,60 & 33,98 \\
48 horas & 2,70 & 32,72 \\
72 horas & 2,70 & 32,72 \\
\hline
\end{tabular}

máxima, de 32,72 Å, pois mesmo com o aumento do tempo reacional o valor $d$ de espaçamento interlamelar não é alterado. Deve-se observar que para 24 horas de reação é obtido um valor $d$ maior, mas, como em tempos superiores o valor volta a ser de exatamente igual a $32,72 \AA$, este pode ser considerado estatisticamente igual. Chama a atenção o reduzido tempo que a PVP necessita para ser intercalada, o qual provavelmente se deve à sua estrutura amídica, que seria capaz de estabilizar as lamelas negativamente carregadas da argila através de ligações eletrostáticas.

Os difratogramas de raios $\mathrm{X}$ dos sistemas polímero - organoargila, na proporção 1:1, em todos os diferentes tempos reacionais testados (15 minutos, 30 minutos, 45 minutos, 1 hora, 24 horas, 48 horas, 72 horas) em diclorometano a temperatura ambiente encontram-se na Figura 3. Os valores de espaçamento interlamelar correspondentes podem ser acompanhados pela Tabela 3. Assim como nas reações anteriores (proporção 2:1), são notadas significativas variações de espaçamento interlamelar em relação ao viscogel B8 puro (25,98 ̊̊), superiores a $6,7 \AA$, a exceção dos 45 minutos, o que parece ser atribuído à técnica, já que os valores tornam a crescer com o aumento do tempo reacional. Portanto, os resultados sugerem a formação do nanocompósito mesmo com a redução da quantidade de polímero utilizada. Ainda, de acordo com os resultados encontrados, pode-se considerar

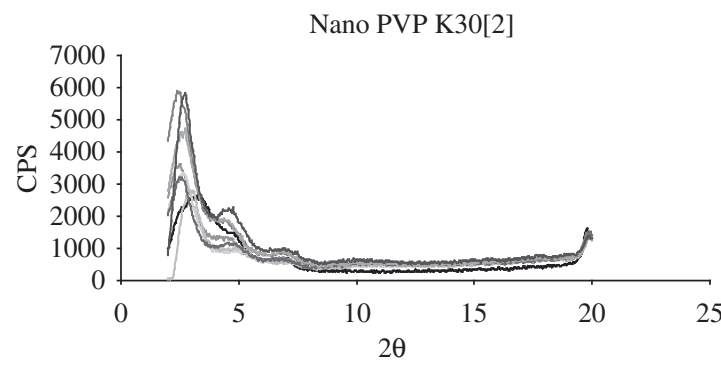

- viscogel B8 - 1p1a 15 minutos - 1p1a 30 minutos
-1p1a 45 minutos - 1p1a 1 hora - 1p1a 24 horas
\[ \text { 1p1a } 48 \text { horas - 1p1a } 72 \text { horas } \]

Figura 3. Difratogramas dos sistemas polímero - organoargila estudados, na proporção $1: 1$. 
Tabela 3. Valores de espaçamento interlamelar calculados para os sistemas polímero - organoargila estudados, na proporção 1:1.

\begin{tabular}{ccc}
\hline $\begin{array}{c}\text { Tempo } \\
\text { reacional }\end{array}$ & Deslocamento $\left(^{\circ}\right)$ & $\begin{array}{c}\text { Espaçamento } \\
\text { interlamelar }(\AA)\end{array}$ \\
\hline viscogel B8 & 3,40 & 25,98 \\
15 minutos & 2,55 & 34,65 \\
30 minutos & 2,60 & 33,98 \\
45 minutos & 3,10 & 28,50 \\
1 hora & 2,55 & 34,65 \\
24 horas & 2,60 & 33,98 \\
48 horas & 2,70 & 32,72 \\
72 horas & 2,70 & 32,72 \\
\hline
\end{tabular}

que 1 hora de reação é suficiente para que seja alcançada a distância interlamelar máxima, de 34,65 ̊.

Os difratogramas de raios $\mathrm{X}$ dos sistemas polímero - organoargila, na proporção 1:2, em todos os diferentes tempos reacionais testados (15 minutos, 30 minutos, 45 minutos, 1 hora, 24 horas, 48 horas, 72 horas) em diclorometano a temperatura ambiente encontram-se na Figura 4. Os valores de espaçamento interlamelar correspondentes podem ser acompanhados pela Tabela 4. Os resultados sugerem a formação do nanocompósito de acordo com os valores de espaçamento interlamelar observados. Pode-se considerar que 1 hora de reação é suficiente para que seja alcançada a distância interlamelar máxima, de 32,72 Å. Vale ressaltar que,

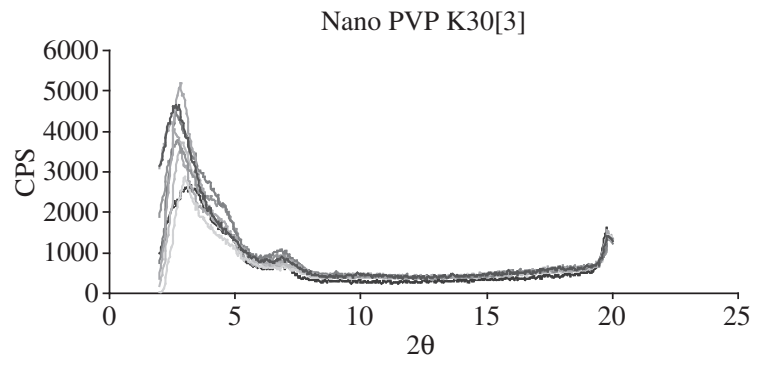

$$
\begin{gathered}
\text { - viscogel B8 -1p2a } 15 \text { minutos - 1p2a } 30 \text { minutos } \\
\text {-1p2a } 45 \text { minutos - 1p2a } 1 \text { hora -1p2a } 24 \text { horas } \\
- \text { 1p2a } 48 \text { horas - 1p2a } 72 \text { horas }
\end{gathered}
$$

Figura 4. Difratogramas dos sistemas polímero - organoargila estudados, na proporção $1: 2$.

Tabela 4. Valores de espaçamento interlamelar calculados para os sistemas polímero - organoargila estudados, na proporção 1:2.

\begin{tabular}{ccc}
\hline $\begin{array}{c}\text { Tempo } \\
\text { reacional }\end{array}$ & Deslocamento $\left(^{\circ}\right)$ & $\begin{array}{c}\text { Espaçamento } \\
\text { interlamelar }(\AA)\end{array}$ \\
\hline Viscogel B8 & 3,40 & 25,98 \\
15 minutos & 2,45 & 36,06 \\
30 minutos & 3,00 & 29,45 \\
45 minutos & 2,95 & 29,95 \\
1 hora & 2,70 & 32,72 \\
24 horas & 2,70 & 32,72 \\
48 horas & 2,85 & 31,00 \\
72 horas & 2,75 & 32,13 \\
\hline
\end{tabular}

em termos numéricos, este não foi o valor mais alto obtido, mas sim o que parece se manter e ainda corroborar os demais resultados.

Já tendo sido relatado em literatura o efeito do solvente sobre o inchamento de organoargilas ${ }^{[21]}$, foi, ainda, preparado um "branco", ou seja, um sistema contendo somente viscogel B8 e diclorometano, com o objetivo de minimizar este efeito, na tentativa de garantir maior confiabilidade aos resultados obtidos. O espaçamento interlamelar do branco foi realmente inferior ao do viscogel B8 quando analisado sem solvente, $21,82 \AA$ vs. $25,98 \AA$, respectivamente, resultado que ratifica a literatura e ressalta ainda mais a confiança a respeito dos resultados da intercalação PVP - organoargila.

\section{PVP K-30 - MMT}

Como a inserção do íon alquilamônio é um recurso para aumentar o espaço interlamelar, auxiliando o processo de intercalação, além de criar um ambiente lipofílico, permitindo a entrada de polímeros lipofílicos, e visto que a PVP não apresenta (de acordo com os resultados obtidos e aliados à sua solubilidade) nenhum destes problemas, apontou para a possibilidade de estudo do seu comportamento com argila não tratada e utilizando-se água para tal. Desse modo, foram estudadas as mesmas condições estabelecidas para PVP viscogel B8.

Os difratogramas de raios $\mathrm{X}$ dos sistemas polímero - argila sódica, na proporção 2:1, em todos os diferentes tempos reacionais testados (15 minutos, 30 minutos, 45 minutos, 1 hora, 24 horas, 48 horas, 72 horas) em água a temperatura ambiente encontram-se na Figura 5. Os valores de espaçamento interlamelar correspondentes podem ser acompanhados pela Tabela 5. Os resultados corresponderam às expectativas, pois, tal qual obtido com o viscogel B8, aumentos nos valores de espaçamento interlamelar foram notórios (superiores a $10 \AA$ ) com a adição da PVP ao sistema. A intercalação deste polímero com a argila sódica em água se passa ainda mais rápido. Com apenas 15 minutos de reação a distância interlamelar máxima (e que parece se manter) é alcançada $(25,99 \AA$ A $)$. A alta afinidade do polímero pela lamela negativa-

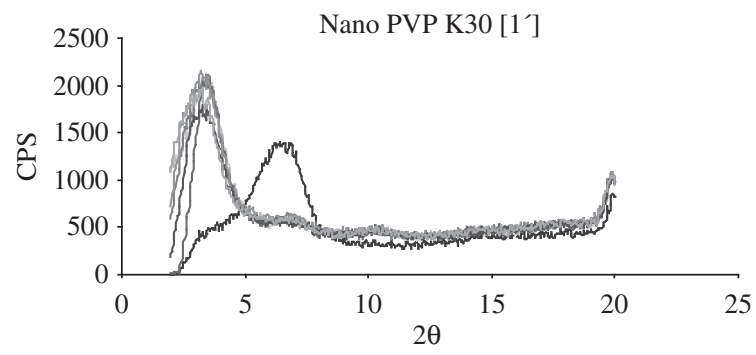

$$
\begin{gathered}
\text { —arg sódica } \quad \text {-2p1as } 15 \text { minutos - 2p1as } 30 \text { minutos } \\
\text {-2p1as } 45 \text { minutos — 2p1as } 1 \text { hora }-2 \text { p1as } 24 \text { horas } \\
\text { - 2p1as } 48 \text { horas }-2 \text { p1as } 72 \text { horas }
\end{gathered}
$$

Figura 5. Difratogramas dos sistemas polímero - argila sódica estudados, na proporção 2:1. 
Tabela 5. Valores de espaçamento interlamelar calculados para os sistemas polímero - argila sódica estudados, na proporção 2:1.

\begin{tabular}{lcc}
\hline $\begin{array}{c}\text { Tempo } \\
\text { reacional }\end{array}$ & Deslocamento $\left(^{\circ}\right)$ & $\begin{array}{c}\text { Espaçamento } \\
\text { interlamelar }(\mathbf{\AA})\end{array}$ \\
\hline Argila sódica & 6,55 & 13,49 \\
15 minutos & 3,40 & 25,99 \\
30 minutos & 3,40 & 25,99 \\
45 minutos & 3,50 & 25,24 \\
1 hora & 3,55 & 24,89 \\
24 horas & 3,20 & 27,61 \\
48 horas & 3,45 & 25,61 \\
72 horas & 3,32 & 26,61 \\
\hline
\end{tabular}

mente carregada da argila, somada ao excesso do mesmo na reação, parecem ser os responsáveis pelos resultados.

Os difratogramas de raios $\mathrm{X}$ dos sistemas polímero - argila sódica, na proporção 1:1, em todos os diferentes tempos reacionais testados (15 minutos, 30 minutos, 45 minutos, 1 hora, 24 horas, 48 horas, 72 horas) em água a temperatura ambiente encontram-se na Figura 6. Os valores de espaçamento interlamelar correspondentes podem ser acompanhados pela Tabela 6. Em vista dos resultados obtidos, a redução da quantidade de polímero utilizada, leva a uma redução da velocidade de intercalação do polímero. Todos os valores de espaçamento interlamelar foram novamente elevados (superiores a $10 \AA$ ) e a distância interlamelar máxima parece ser conseguida com 1 hora de reação $(26,37 \AA)$.

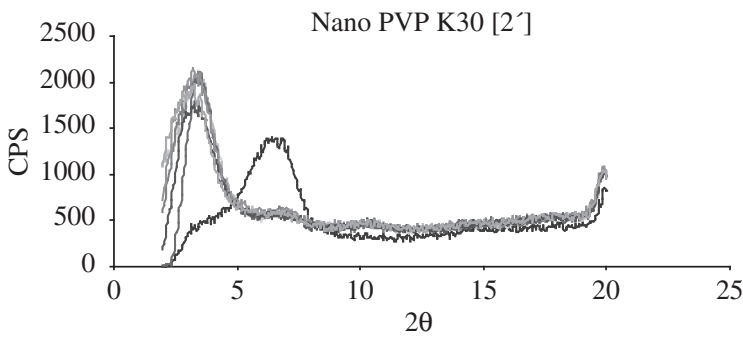

\begin{tabular}{|c|} 
- arg sódica $-1 \mathrm{p} 1$ as 15 minutos $-1 \mathrm{p} 1$ as 30 minutos \\
$-1 \mathrm{p} 1$ as 45 minutos $-1 \mathrm{p} 1$ as 1 hora $-1 \mathrm{p} 1$ as 24 horas \\
- 1p1as 48 horas $-1 \mathrm{p} 1$ as 72 horas
\end{tabular}

Figura 6. Difratogramas dos sistemas polímero - argila sódica estudados, na proporção 1:1.

Tabela 6. Valores de espaçamento interlamelar calculados para os sistemas polímero - argila sódica estudados, na proporção 1:1.

\begin{tabular}{lcc}
\hline $\begin{array}{c}\text { Tempo } \\
\text { reacional }\end{array}$ & Deslocamento $\left(^{\circ}\right)$ & $\begin{array}{c}\text { Espaçamento } \\
\text { interlamelar }(\AA)\end{array}$ \\
\hline Argila sódica & 6,55 & 13,49 \\
15 minutos & 3,55 & 24,86 \\
30 minutos & 3,35 & 26,37 \\
45 minutos & 3,60 & 24,64 \\
1 hora & 3,35 & 26,37 \\
24 horas & 3,30 & 26,78 \\
48 horas & 3,35 & 26,37 \\
72 horas & 3,35 & 26,37 \\
\hline
\end{tabular}

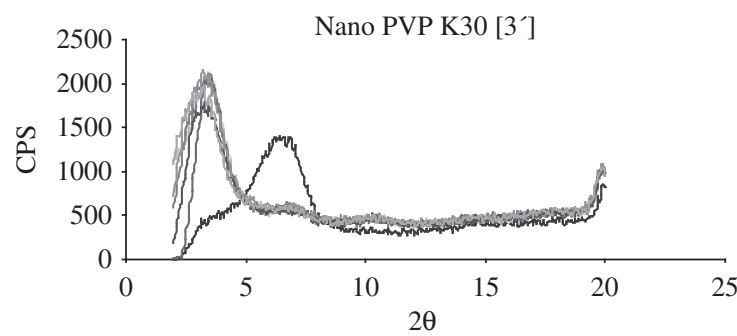

$$
\begin{gathered}
\text { - arg sódica }-1 \mathrm{p} 2 \text { as } 15 \text { minutos }-1 \mathrm{p} 2 \text { as } 30 \text { minutos } \\
\text {-1p2as } 45 \text { minutos -1p2as } 1 \text { hora }-1 \mathrm{p} 2 \text { as } 24 \text { horas } \\
\text { - 1p2as } 48 \text { horas - 1p2as } 72 \text { horas }
\end{gathered}
$$

Figura 7. Difratogramas dos sistemas polímero - argila sódica estudados, na proporção 1:2.

Tabela 7. Valores de espaçamento interlamelar calculados para os sistemas polímero - argila sódica estudados, na proporção 1:2.

\begin{tabular}{lcc}
\hline $\begin{array}{c}\text { Tempo } \\
\text { reacional }\end{array}$ & Deslocamento $\left(^{\circ}\right)$ & $\begin{array}{c}\text { Espaçamento } \\
\text { interlamelar }(\mathbf{A})\end{array}$ \\
\hline Argila sódica & 6,55 & 13,49 \\
15 minutos & 3,70 & 23,88 \\
30 minutos & 3,70 & 23,88 \\
45 minutos & 3,70 & 23,88 \\
1 hora & 3,55 & 24,89 \\
24 horas & 3,55 & 24,89 \\
48 horas & 3,50 & 25,24 \\
72 horas & 3,60 & 24,64 \\
\hline
\end{tabular}

Os difratogramas de raios $\mathrm{X}$ dos sistemas polímero - argila sódica, na proporção 1:2, em todos os diferentes tempos reacionais testados (15 minutos, 30 minutos, 45 minutos, 1 hora, 24 horas, 48 horas, 72 horas) em água a temperatura ambiente encontram-se na Figura 7. Os valores de espaçamento interlamelar correspondentes podem ser acompanhados pela Tabela 7 .

Os resultados parecem reproduzir o encontrado para a proporção 1:1, tanto quanto à evidência dos valores de espaçamento interlamelar (superiores a $10 \AA$ ) , quanto à distância interlamelar máxima, em 1 hora de reação $(24,89 \AA$ A).

Ainda, todos os melhores pontos obtidos de cada condição reacional tiveram suas reações repetidas, porém com um intumescimento prévio das argilas (por 1 hora em seus respectivos solventes) antes da adição da PVP, e novamente avaliadas por DRX. Em todos os casos, este intumescimento não trouxe mudanças significativas dos valores de espaçamento interlamelar.

\section{Conclusões}

Por meio deste estudo foi possível não somente elucidar a formação dos nanocompósitos PVP K-30 - viscogel B8 e PVP K-30 - argila sódica, como avaliar seu processo reacional, o qual se dá em tempo reduzido (em até 1 hora de reação) provavelmente devido à estrutura amídica do polímero em questão. Este seria capaz de estabilizar, por meio de ligações eletrostáticas, as lamelas negativamente carregadas da argila. 
O sistema PVP K-30 - argila sódica ainda teria como vantagens a não necessidade de tratamento prévio da argila e o uso da água como solvente.

\section{Agradecimentos}

Ao apoio financeiro fornecido pelo CNPq e CAPES.

\section{Referências Bibliográficas}

1. Minatech Information Day. Conference on Micro and Nano Technologies. A World Wide view. AIRI - Associazione Italiana per la Ricerca Industriale. European Reports on Technologies, Italy (2001).

2. Van Savage, G. \& Rhodes, C. T. - Drug Dev. Ind. Pharm., 21, p. 93 (1995).

3. Desphpande, A. A.; Rhodes, C. T. \& Shah, N. H. - Drug Dev. Ind. Pharm., 22, p. 531 (1996).

4. Chien, Y. W. - Med. Prog. Technol., 15 (21) (1989a).

5. Chien, Y. W. "Novel Drug Systems", Marcel Dekker Inc., New York (1992b).

6. Vieweg, R. et al. Kunststoff-Handbuch XI, Carl-HanserVerlag, Munich (1971).

7. Ullmann's Encyclopedia of Industrial Chemistry, WileyVCH, 21, p. 752-754, (1992). Disponível em: <http:// www.wiley-vch.de/vch/software/ullmann/index. html>. Acesso em: 15 dez. 2006.

8. Kibbe, A. H. - "Handbook of Pharmaceutical Excipients", $3^{\text {rd }}$ ed., p. 440 (2000a).

9. GAF(ISP) Technical Bulletin 2302-203 SM-1290 - "PVP polyvinylpyrrolidone Polymers" (1990).

10. Kristensen A. B.; Faramarzi J. \& Krejberg, T. - WO2005061689, 2005.
11. Murphy, T. K. \& Eisenhart, E. K. - WO-2005102272 (2005).

12. Rudnic, E. M. \& Schwartz, J. D. - "Remington: The Science and Practice of Pharmacy", Lippincott Williams \& Wilkins, p. 858-893 (2000).

13. Murray, H. H. - Appl. Clay Sci., 17, 5-6, p. 207-221 (2000).

14. Carretero, M. I. - Appl. Clay Sci., 21, p. 155-163 (2002).

15. Galan, E.; Liso, M. J. \& Forteza, M. - Bol. Soc. Esp. Miner., 8, p. 369-378, (1985).

16. Damour, A. \& Salvetat, D. - Ann. Chim. Phys., 3 (21), p. 376-383 (1847).

17. Giannelis, E. P. - Adv. Mater., 8 (1), p. 29-35 (1996).

18. Dornelas, CB. - "Preparação e caracterização de novos nanocompósitos de PVP - montmorilonita organofílica como excipiente farmacêutico", Dissertação de Mestrado, Universidade Federal do Rio de Janeiro, Brasil (2006).

19. Coutinho, S. S. - "Estudo do desenvolvimento de comprimidos de liberação prolongada de blendas polissacarídicas de montimorilonita. Avaliação do uso de monoestearato de glicerila, quitosano e HPMC", Dissertação de Mestrado, Universidade Federal do Rio de Janeiro, Brasil (2004).

20. Katz, H. S. \& Milewski, J. W. - "Handbook of Fillers for plastics", Van Nostrand Reinhold, New York (1987).

21. Burgentzlé, D.; Duchet, J.; Gérard, J. F.; Jupin, A. \& Fillon, B. - J. Colloid Interface Sci, 278, p. 26-39 (2004).

Enviado: 06/08/07

Reenviado: $16 / 02 / 08$

Aceito: $29 / 02 / 08$ 